EXPERIMENTAL STUDY

\title{
Bisphenol-A, an environmental estrogen, activates the human orphan nuclear receptor, steroid and xenobiotic receptor-mediated transcription
}

\author{
Akira Takeshita, Noriyuki Koibuchi ${ }^{1}$, Junko Oka, Manabu Taguchi, Yoshimasa Shishiba and Yasunori Ozawa \\ Division of Endocrinology and Metabolism, Toranomon Hospital, Okinaka Memorial Institute for Medical Research, Tokyo 105-8470, Japan and \\ ${ }^{1}$ Department of Physiology, Gunma University School of Medicine and CREST, Japan Science and Technology Corporation, Maebashi, Gunma 371-8511, Japan \\ (Correspondence should be addressed to Akira Takeshita, Division of Endocrinology and Metabolism, Toranomon Hospital, Okinaka Memorial Institute for \\ Medical Research, 2-2-2 Toranomon, Minato, Tokyo 105-8470, Japan; Email: coactivator@mac.com)
}

\begin{abstract}
Background: There is increasing concern about endocrine-disrupting chemicals (EDCs) which may produce adverse health effects in humans and other species. One such chemical, bisphenol-A (BPA), a monomer of polycarbonate plastics, is widely used in consumer products; it has been reported to contain estrogenic activity through binding to estrogen receptors. Cytochrome P450 monooxygenase 3A4 (CYP3A4) is one of the key enzymes for the metabolism of endogenous steroids and foreign chemicals in liver. The orphan nuclear receptor, steroid and xenobiotic receptor (SXR/PXR), has recently been isolated. A variety of known inducers of CYP3A4 bind to SXR/PXR, and stimulate transcription on xenobiotic-response elements (XREs) located in the promoter region of the CYP3A4 gene. Recent study has shown that EDCs, diethylhexylphthalate (DEHP) and nonylphenol, but not $\mathrm{BPA}$, induce mouse SXR/PXR-mediated transcription. However, it is known that species differences in SXR alter CYP3A inducibility.

Objective: To test whether BPA stimulates human SXR/PXR-mediated transcription using reporter gene assays.

Methods: Transfection assays were performed with human SXR/PXR expression plasmid and a reporter plasmid containing the XREs in the CYP3A4 gene promoter in HepG2 cells. BPA-induced interaction of human SXR/PXR with steroid receptor coactivator-1 (SRC-1) was analyzed by mammalian two-hybrid assays.

Results: BPA, as well as DEHP, activated human SXR-mediated transcription on the XREs. In mammalian two-hybrid assays, BPA recruited SRC-1 to the ligand-binding domain of human SXR/PXR.

Conclusions: Our observations have indicated that BPA may be a human-specific inducer of the CYP3A4 gene, and may influence the metabolism of endogenous steroids, drugs, and other xenobiotics.
\end{abstract}

European Journal of Endocrinology 145 513-517

\section{Introduction}

There is increasing concern about endocrine-disrupting chemicals (EDCs) which may produce adverse health effects in humans and other species. One such chemical, bisphenol-A (BPA), a monomer of polycarbonate plastics, is widely used in consumer products. For instance, a significant amount of BPA is released into food and human saliva from the inner coating of food cans (1) and dental composites (2) respectively. BPA has a high homology with diethylstilbestrol (DES), a synthetic substance with potent estrogenic activity. Although the affinities of BPA to human estrogen receptor (ER) $\alpha$ and $\beta$ are much lower than those of estradiol and DES, BPA binds to the ERs and stimulates transcription (3), indicating that BPA may act as an environmental estrogen. In fact, BPA induces progesterone receptor expression in the ER-positive human breast cancer cell line, MCF-7 cells, and also increases its proliferation (4).

The cytochrome P450 (CYP) superfamily consists of heme-containing mono-oxygenases which play an important role in the oxidative metabolism of endogenous substances, natural compounds, and xenobiotics. The CYP mono-oxygenase 3A4 (CYP3A4) gene product is the most abundant CYP that is expressed in human liver and it is involved in the metabolism of most drugs, a variety of endogenous steroids, and environmental procarcinogens (5). The expression of CYP3A4 is transcriptionally activated by many natural 
and xenobiotic compounds. For instance, the antibiotic rifampicin is a well-known inducer of the human CYP3A4 gene. Recently, the orphan nuclear receptor, steroid and xenobiotic receptor (SXR) (also called pregnane $X$ receptor (PXR)), has been isolated (6-9). A variety of known inducers of CYP3A4 bind to SXR/ PXR, and stimulate transcription on xenobioticresponse elements (XREs), located in the promoter region of the CYP3A4 gene (6-10).

The SXR/PXR-mediated transactivation by EDCs has recently been reported (11). Diethylhexylphthalate (DEHP) and nonylphenol, but not BPA, induce mouse SXR/PXR-mediated transcription (11). However, there are markedly different activation profiles in response to xenobiotics among species $(7,8)$. For instance, rifampicin is a strong agonist in human and rabbit SXR/PXR, but not in rat and mouse SXR/PXR (12). The differences in xenobiotic actions among species are likely due to differences in the structure of SXR/PXR. Human and mouse SXR/PXR share only $77 \%$ amino acid identity in their ligand-binding domain (LBD), whereas they share $96 \%$ identity in the DNA-binding domain (DBD) (12). Thus, human SXR/PXR may possess different inducibility from mouse SXR/PXR by EDCs.

In the present study, we tested whether BPA stimulates human SXR/PXR-mediated transcription to induce the CYP3A4 gene.

\section{Materials and methods}

\section{Reagents and chemicals}

Rifampicin, corticosterone, and DEHP were obtained from Sigma Chemical Co. (St Louis, MO, USA). BPA was from Wako Pure Chemical Industries Ltd (Osaka, Japan).

\section{Plasmids}

Human SXR in pCDG1 was kindly provided by Dr R M Evans, Salk Institute, La Jolla, CA, USA (8). A schematic diagram of GAL4 or VP16 fusion constructs used is shown in Fig. 1. GAL4 SXR-LBD and GAL4 SRC1-RID were constructed by ligating the LBD of human SXR (amino acids 107-434) and nuclear receptor interacting domain (RID) containing three LXXLL motifs in human steroid receptor coactivator-1 (SRC-1) (amino acids 595-780) (13) into the GAL4 DBD in the pM expression vector (Clontech, Palo Alto, CA, USA) respectively. VP16 SXR-LBD and VP16 SRC-1-RID were constructed by ligating the same amino acid fragments of SXR and SRC-1 in GAL4 SXR-LBD and GAL4 SRC-1RID into downstream of the VP16 activation domain in AASV-VP16 (kindly provided by Dr S M Weissman, Yale School of Medicine, New Haven, CT, USA) (14) respectively. The chimeric CYP3A4 luciferase (LUC)
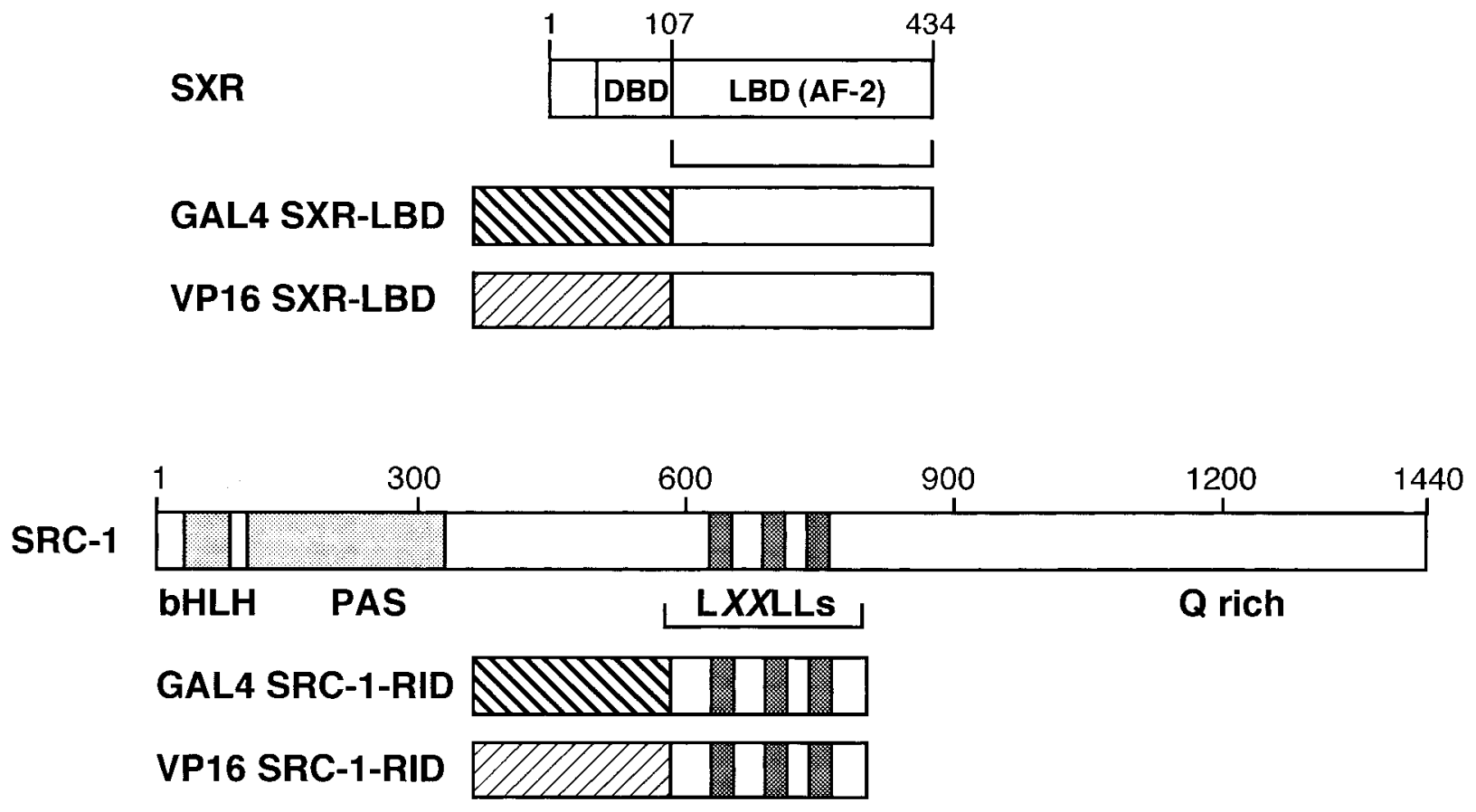

Figure 1 Schematic diagram of GAL4 or VP16 constructs used in transfection reporter assays. GAL4 SXR-LBD and VP16 SXR-LBD contain residues 107-434 of human SXR. GAL4 SRC-1-RID and VP16 SRC-1-RID contain residues 595-780 of human SRC-1. The fragment of SRC-1 contains three consensus $L X X L L$ motifs, which are important for ligand-dependent interaction with nuclear hormone receptors. AF-2, ligand-dependent transactivation domain; bHLH, basic helix loop helix; PAS, Per Arnt Sim; Q, glutamine.

www.eje.org 
reporter construct, a xenobiotic-responsive enhancer module (XREM)-CYP3A4-LUC, containing the enhancer (nucleotides -7836 to -7208 ) and promoter (nucleotides -362 to +53 ) of human CYP3A4 driving luciferase expression, was kindly provided by Dr S A Kliewer, Glaxo Wellcome Research and Development, Research Triangle Park, NC, USA (10, 15). The LUC reporter construct, $5 \times$ upstream activating sequence (UAS)-thymidine kinase minimum promoter (TK)-LUC, was kindly provided by Dr A N Hollenberg, Beth Israel Deaconess Medical Center, Boston, MA, USA (16).

\section{Transient cotransfection experiments}

HepG2 cells or CV-1 cells were grown in Dulbecco's modified Eagle's medium and 5\% fetal calf serum. The serum was stripped of hormones by constant mixing with 5\% (w/v) AG1-X8 resin (Bio-Rad, Hercules, CA, USA) and powdered charcoal before ultrafiltration. The cells were maintained without antibiotics. Cells were transiently transfected using the calcium phosphate coprecipitation method in six-well plates with $1.5 \mu \mathrm{g}$ reporter plasmid containing XREM-CYP3A4-LUC or $5 \times$ UAS-TK-LUC cDNA with expression vectors as indicated in the Figure legends. CMV- $\beta$-galactosidase plasmid was used as an internal control. In some samples, empty expression vectors were added to equalize total transfected plasmid concentration. Cells were grown for $24 \mathrm{~h}$ in the absence or presence of ligand, and then harvested. Cell extracts were analyzed for both luciferase and $\beta$-galactosidase activity in order to correct for transfection efficiency as previously described (17). The corrected luciferase activities of untreated samples were normalized to the luciferase activities of samples as described in the Figure legends. All transfection studies were repeated at least twice in triplicate. The results shown are the means \pm S.D. $(n=3)$.

\section{Results}

To determine whether BPA stimulates human SXR/ PXR-mediated transcription, transient transfection assays were performed with human SXR/PXR expression plasmid and a reporter plasmid, XREM-CYP3A4LUC, containing the enhancer and promoter of CYP3A4 driving luciferase gene expression (10, 15) in a human liver-derived cell line, HepG2 (Fig. 2). Unlike the mouse SXR/PXR system (11), human SXR/ PXR-mediated transcription was stimulated by BPA on the CYP3A4 gene. Thus, induction of the CYP3A4 gene by BPA was species-specific. DEHP treatment showed similar transcriptional efficacy. Of note, compared with rifampicin and corticosterone, which were efficacious activators of human SXR/PXR, these two EDCs had significant effects only at a high concentration $(>1 \mu \mathrm{M})$.

Transcriptional activation by nuclear hormone receptors (NRs) is mediated by ligand-dependent

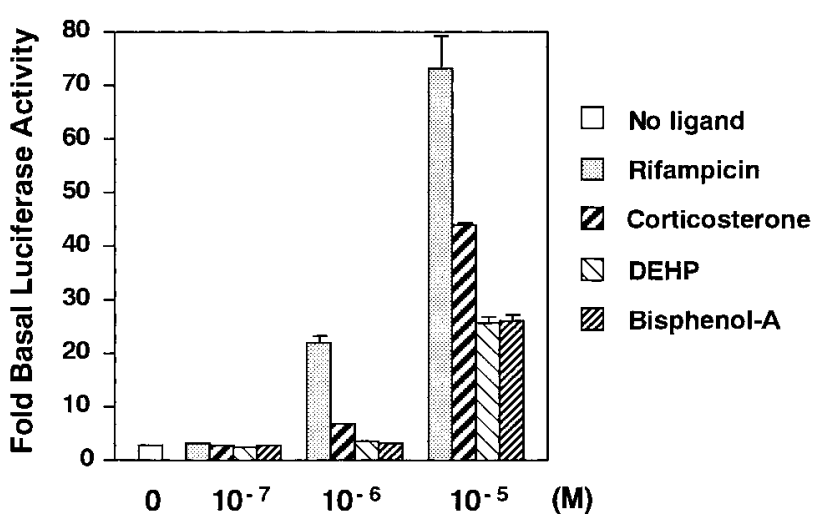

Figure 2 BPA stimulates human SXR-mediated transcription on the CYP3A4 promoter. The expression plasmid containing human SXR $(0.1 \mu \mathrm{g})$ and the reporter plasmid XREM-CYP3A4LUC $(1.5 \mu \mathrm{g})$ were cotransfected with CMV- $\beta$-galactosidase control vector $(0.1 \mu \mathrm{g})$ in HepG2 cells. Cells were treated with several compounds for $24 \mathrm{~h}$ and analyzed for luciferase activity. Luciferase activity was normalized to $\beta$-galactosidase activity and then calculated as fold luciferase activity with 1-fold basal activity defined as the luciferase activity with human SXR in the absence of the ligand. The results are expressed as means \pm S.D. $(n=3)$. Dose-response analysis was performed with rifampicin, corticosterone, DEHP, and BPA.

interaction with coactivators. SRC-1 belongs to a $160 \mathrm{kDa}$ subset of the nuclear receptor coactivator family that includes TIF2/GRIP1 and TRAM-1/AIB1/ RAC3/ACTR $(18,19)$. SRC-1 binds to a variety of NRs in a ligand-dependent manner and enhances ligandinduced transcriptional activity of NRs. To determine whether BPA increases the interaction of human SXR/ PXR with SRC-1, mammalian two-hybrid assays were performed. As illustrated in Fig. 1, the LBD of human SXR/PXR was fused to the DBD of GAL4 (GAL4 SXRLBD), and the RID of SRC-1 containing three LXXLL motifs was fused to the transactivation domain of VP16 (VP16 SRC-1-RID). These constructs were cotransfected with a reporter plasmid containing five copies of a GAL4 UAS ( $5 \times$ UAS-TK-LUC) in CV- 1 cells. When the transfection was performed with a combination of GAL4 SXR-LBD and VP16 SRC-1-RID, both rifampicin and BPA strongly enhanced the reporter activities, compared with a combination of GAL4 SXR-LBD and empty VP16 vector (Fig. 3A). The assay was also performed using the opposite configuration (Fig. 3B). GAL4 SRC-1-RID interacted with VP16 SXR-LBD in the presence of rifampicin and BPA. Similar results were obtained when we used HepG2 cells, instead of CV-1 cells. These mammalian two-hybrid assays indicated that BPA, as well as rifampicin, recruits SRC-1 to the LBD of human SXR/PXR.

\section{Discussion}

Previous studies have emphasized only the estrogenic activity of BPA (20). We have here shown another 
A

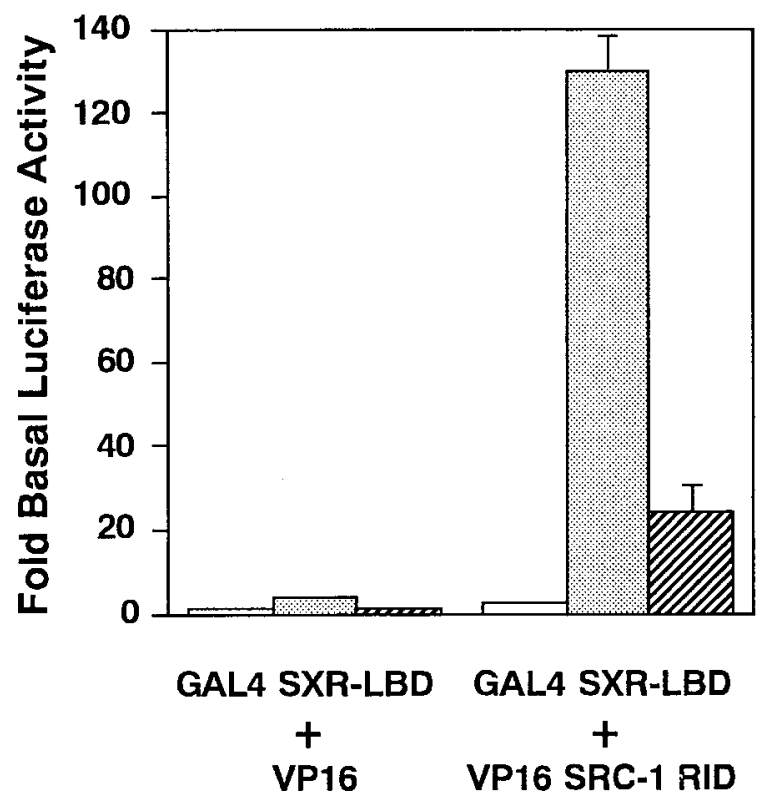

B

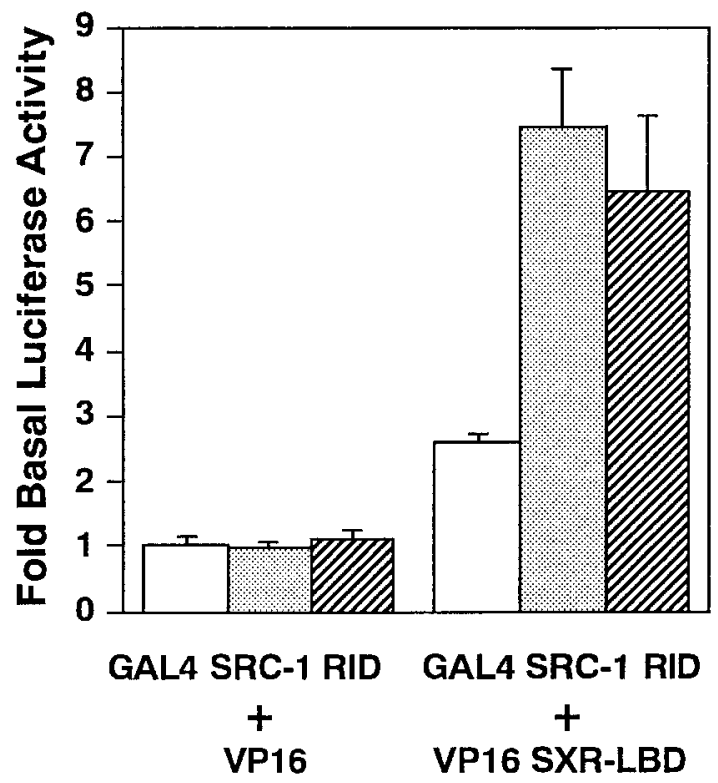

Figure 3 In vivo interaction between LBD of human SXR and RID of SRC-1. The expression plasmids encoding GAL4 SXR-LBD $(0.1 \mu \mathrm{g})$ and either VP16 alone or VP16 SRC-1-RID $(0.5 \mu \mathrm{g})(\mathrm{A})$ or the plasmids encoding GAL4 SRC-1-RID $(0.1 \mu \mathrm{g})$ and either VP16 alone or VP16 SXR-LBD $(0.5 \mu \mathrm{g})(\mathrm{B})$ were cotransfected with $5 \times$ UAS-TK-LUC reporter plasmid $(1.5 \mu \mathrm{g})$ and CMV- $\beta$ galactosidase control vector $(0.1 \mu \mathrm{g})$ in CV-1 cells. Cells were treated with $10^{-5} \mathrm{M}$ rifampicin or BPA for $24 \mathrm{~h}$. The corrected luciferase activity was calculated as fold luciferase activity with 1-fold basal activity defined as the luciferase activity with GAL4 SXRLBD and VP16 in the absence of the ligand. The results are expressed as means \pm S.D. $(n=3)$. Open bars, no ligand; shaded bars, rifampicin; hatched bars, BPA.

function of BPA as a ligand of human SXR/PXR. BPA activates human SXR on a reporter gene containing XREs in the CYP3A4 gene promoter. The response to BPA is mediated by the LBD of human SXR, which contains ligand-dependent transactivation domain (AF-2). In addition, BPA recruits SRC-1, a nuclear receptor coactivator, to the LBD of human SXR. Since CYP3A4 plays a critical role in the catalysis of steroid hormones, BPA may influence the metabolism of endogenous steroids such as corticosteroids and gonadal steroids in adults, children, and fetuses.

The induction of CYP3A expression by xenobiotics is quite different between humans and rodents $(5,7,8)$. Recent studies have revealed that divergent structure in the LBD of SXR/PXR causes marked pharmacological differences in SXR/PXR activation profiles (12). Although BPA failed to induce mouse SXR/PXRmediated transcription in a previous report (11), it stimulated human SXR/PXR-mediated transcription in the present study. Thus, the effects of EDCs on CYP3A expression are species-specific. Xie et al. (21) created a humanized xenobiotic response in mice by replacing with the human SXR/PXR gene. Such an approach will be useful in addressing the effects of EDCs on human CYP3A expression.
Whether a low dose of exposure to environmental agents can influence human endocrine functions is a common concern about all EDCs. In our in vitro reporter assay, both BPA and DEHP induced human SXR/PXR-mediated transcription only at high concentrations. Although these EDCs showed low potencies in vitro, we suggest several possibilities that BPA and/or DEHP may function as EDCs to influence human SXRmediated transcription in vivo. First, the potency of an in vitro reporter assay may be less than that of the situation in vivo. Steinmetz et al. (22) studied the estrogenic potency of BPA in vivo and in vitro. They observed that BPA had a similar efficacy to estradiol in inducing hyperprolactinomas in F344 rats, while BPA was 1000- to 5000-fold less active than estradiol in vitro. Secondly, combinations of BPA with other xenobiotics such as DEHP may additively activate human SXR/PXR. To support this hypothesis, BPA was detected along with DEHP, nonylphenol, and di- $n$ butylphthalate in dialysate in plastic dialysis bags (23). In addition, Blumberg et al. (8) reported that the cocktails of endogenous steroids additively increase human SXR-mediated transcription. Thirdly, we may be exposed to significant amounts of BPA and/or DEHP. For instance, 90-931 $\mu \mathrm{g}$ BPA was detected in the 
saliva of patients treated with dental sealants (2), and 1.3-17200 $\mu \mathrm{g} / \mathrm{l}$ BPA with a median concentration of $269 \mu \mathrm{g} / \mathrm{l}$ in hazardous waste landfill leachates was collected in Japan (24). As much as 44.3-197.1 mg DEHP was extracted from the dialyzer during a single dialysis session (25). Therefore, BPA and/or DEHP may influence human CYP3A expression in certain circumstances.

We have not tested other environmental estrogens such as polychlorinated hydroxybiphenyls, DDT and its derivatives, and phytoestrogens. It will be important to know whether those xenoestrogens activate human SXR/PXR.

In summary, our study suggests that BPA, as well as DEHP, acts as a ligand of human SXR/PXR. BPA may be a human-specific inducer of the CYP3A4 gene, and may potentially influence the metabolism of endogenous steroids, drugs, and other xenobiotics.

\section{Acknowledgements}

We thank Drs S M Weissman, R M Evans, A N Hollenberg, and S A Kliewer for providing plasmids and $\mathrm{H}$ Sato (Dokkyo University, Japan) for helpful discussion.

\section{References}

1 Brotons JA, Olea-Serrano MF, Villalobos M, Pedraza V \& Olea N. Xenoestrogen released from lacquer coatings in food cans. Environmental Health Perspectives 1995103 608-612.

2 Olea N, Pulgar R, Perez P, Olea-Serrano F, Rivas A, NovilloFertrell A et al. Estrogenicity of resin-based composites and sealants used in dentistry. Environmental Health Perspectives 1996 104 298-305.

3 Kuiper GG, Lemmen JG, Carlsson B, Corton JC, Safe SH, van der Saag PT et al. Interaction of estrogenic chemicals and phytoestrogens with estrogen receptor $\beta$. Endocrinology 1998 $1394252-4263$.

4 Krishnan AV, Stathis P, Permuth SF, Tokes L \& Feldman D. Bisphenol-A: an estrogenic substance is released from polycarbonate flasks during autoclaving. Endocrinology 1993132 2279-2286.

5 Waxman DJ. P450 gene induction by structurally diverse xenochemicals: central role of nuclear receptors CAR, PXR, and PPAR. Archives of Biochemistry and Biophysics 1999369 11-23.

6 Kliewer SA, Moore JT, Wade L, Staudinger JL, Watson MA, Jones SA et al. An orphan nuclear receptor activated by pregnanes defines a novel steroid signaling pathway. Cell 1998 $9273-82$

7 Lehmann JM, Mckee DD, Watson MA, Wilson TM, Moore JT \& Kliewer SA. The human orphan nuclear receptor PXR is activated by compounds that regulate CYP3A4 gene expression and cause drug interactions. Journal of Clinical Investigation 1998 102 1016-1023.

8 Blumberg B, Sabbagh W Jr, Juguilon H, Bolado J Jr, van Meter CM, Ong ES et al. SXR, a novel steroid and xenobioticsensing nuclear receptor. Genes and Development 199812 31953205.
9 Bertilsson G, Heidrich J, Svensson K, Asman M, Jendeberg L, Sydow-Backman M et al. Identification of a human nuclear receptor defines a new signaling pathway for CYP3A induction. PNAS $19989512208-12213$.

10 Goodwin B, Hodgson E \& Liddle C. The orphan human pregnan X receptor mediates the transcriptional activation of CYP3A4 by rifampicin through a distal enhancer module. Molecular Pharmacology 199956 1329-1339.

11 Masuyama H, Hiramatsu Y, Kunitomi M, Kudo T \& MacDonald PN. Endocrine disrupting chemicals, phthalic acid and nonylphenol, activate pregnane $\mathrm{X}$ receptor-mediated transcription. Molecular Endocrinology 2000 14 421-428.

12 Jones SA, Moore LB, Shenk JL, Wisely GB, Hamilton GA, McKee DD et al. The pregnane $\mathrm{X}$ receptor: a promiscuous xenobiotic receptor that has diverged during evolution. Molecular Endocrinology 200014 27-39.

13 Takeshita A, Yen PM, Misiti S, Cardona GR, Liu Y \& Chin WW. Molecular cloning and properties of a full-length putative thyroid hormone receptor coactivator. Endocrinology $19961373594-$ 3597.

14 Vasavada HA, Ganguly S, Germino FJ, Wang ZX \& Weissman SM. A contingent replication assay for the detection of proteinprotein interactions in animal cells. PNAS $1991 \mathbf{8 8} 10686-$ 10690.

15 Moore LB, Parks DJ, Jones SA, Bledsoe RK, Consler TG, Stimmel JB et al. Orphan nuclear receptors constitutive androstane receptor and pregnane $X$ receptor share xenobiotic and steroid ligands. Journal of Biological Chemistry 2000275 15122-15127.

16 Cohen RN, Putney A, Wondisford FE \& Hollenberg AN. The nuclear corepressors recognize distinct nuclear receptor complexes. Molecular Endocrinology 200014 900-914.

17 Takeshita A, Cardona GR, Koibuchi N, Suen CS \& Chin WW. TRAM-1, a novel 160-kDa thyroid hormone receptor activator molecule, exhibits distinct properties from steroid receptor coactivator-1. Journal of Biological Chemistry $1997 \quad 272$ 27629-27634.

18 Glass CK \& Rosenfeld MG. The coregulator exchange in transcriptional functions of nuclear receptors. Genes and Development $200014121-141$.

19 McKenna NJ, Lanz RB \& O'Malley BW. Nuclear receptor coregulators: cellular and molecular biology. Endocrine Reviews $199920321-344$.

20 Ben-Jonathan N \& Steinmetz R. Xenoestrogens: the emerging story of bisphenol A. Trends in Endocrinology and Metabolism 1998 $9124-128$.

21 Xie W, Barwick JL, Downes M, Blumberg B, Simon CM, Nelson MC et al. Humanized xenobiotic response in mice expressing nuclear receptor SXR. Nature $2000 \mathbf{4 0 6} 435-439$.

22 Steinmetz R, Brown NG, Allen DL, Bigsby RM \& Ben-Jonathan N. The environmental estrogen bisphenol A stimulates prolactin release in vitro and in vivo. Endocrinology 1997138 1780-1786.

23 Sugimura K, Naganuma T, Kakiya Y, Okada C, Sugimura T \& Kishimoto T. Endocrine-disrupting chemicals in CAPD dialysate and effluent. Blood Purification 200119 21-23.

24 Yamamoto T, Yasuhara A, Shiraishi H \& Nakasugi O. Bisphenol A in hazardous waste landfill leachates. Chemosphere 200142 415-418.

25 Faouzi MA, Dine T, Gressier B, Kambia K, Luyckx M, Pagniez D et al. Exposure of hemodialysis patients to di-2-ethylhexyl phthalate. International Journal of Pharmacology $1999 \mathbf{1 8 0}$ 113-121.

Received 23 January 2001

Accepted 8 May 2001 